CYSTIC FIBROSIS

\title{
Individualised unsupervised exercise training in adults with cystic fibrosis: a 1 year randomised controlled trial
}

\author{
A J Moorcroft, M E Dodd, J Morris, A K Webb
}

Thorax 2004;59:1074-1080. doi: 10.1136/thx.2003.015313

See end of article for authors' affiliations

.....................

Correspondence to: Professor A K Webb, Manchester Adult CF

Centre, South Manchester University Hospitals NHS

Trust, Wythenshawe

Hospital, Manchester M23

9LT, UK; the5webbs@

hotmail.com

Received 26 August 2003 Accepted 22 July 2004
Background: Short term studies of exercise training have shown benefits in cystic fibrosis. Transferring exercise programmes to the community and sustaining them long term is a challenge for the patient. The effectiveness of an individualised unsupervised home based exercise programme was examined in adults with cystic fibrosis over a 1 year period.

Methods: Subjects were randomised to undertake three sessions per week of upper and lower body exercise based on individualised preferences $(n=30)$ or to a control group $(n=18)$. They were evaluated at baseline and at 12 months. The primary outcome measure was improved fitness as assessed by change in blood lactate concentration at the end of an identical constant work rate for both arm and leg ergometric testing. Secondary outcome measurements were heart rate and pulmonary function.

Results: For leg exercise, significant differences were seen at 12 months between the active and control groups in the mean (SE) change in blood lactate levels $(-0.38(0.23) \mathrm{mmol} / \mathrm{l} v 0.45(0.25) \mathrm{mmol} / \mathrm{l}$, $\mathrm{p}<0.05)$ and heart rate $(-4.8(2.5) \mathrm{bpm} v 3.4(2.5) \mathrm{bpm}, \mathrm{p}<0.05)$, confirming a training effect. For arm ergometry there was no change in lactate levels at 12 months but there was a significant difference in forced vital capacity (46 (72) $\mathrm{ml} v-167$ (68) $\mathrm{ml}, \mathrm{p}<0.05)$.

Conclusions: A training effect, as measured by a reduction in lactate levels and heart rate, can be achieved with unsupervised individualised home exercise in adults with cystic fibrosis. A benefit to pulmonary function was observed and together these findings suggest that exercise programmes should be encouraged as an important component of care in cystic fibrosis.
$\mathrm{P}$ eak exercise performance is reduced in patients with cystic fibrosis (CF). The degree of impairment is related to disease severity and correlates with pulmonary function, ${ }^{12}$ nutritional status, ${ }^{3}{ }^{4}$ and peripheral muscle strength. ${ }^{5}$

Exercise training has been studied as a means of improving impaired performance in patients with CF. Several studies have observed an increase in peak exercise capacity in response to programmes of aerobic exercise training; ${ }^{6-8}$ increased strength, ${ }^{9}$ increased sputum expectoration, ${ }^{7}$ decreased breathlessness ${ }^{10}$ and improved ability to perform activities of daily living ${ }^{8}$ have also been demonstrated as a result of exercise training. Data derived from exercise studies also suggest that lung volumes might be improved or preserved. ${ }^{6}{ }^{11-13}$ The recent publication of a 3 year randomised controlled trial of exercise in children which observed a slower rate of annual decline in pulmonary function is therefore of particular importance. ${ }^{14}$ If long term exercise is found to preserve lung function, it would have important implications for survival and would prioritise exercise on the list of treatment modalities for both children and adults with CF.

Most published studies of exercise training in CF have been short term, uncontrolled, and have involved small numbers of subjects. Those studies reporting a training effect have tended to include supervised and hospital based training sessions. Transferring exercise training regimens to the community and sustaining them for long periods of time is a challenge. Failure to continue with regular exercise training has been identified as a problem during unsupervised programmes $^{15}$ and at the end of supervised exercise programmes in children. ${ }^{16}{ }^{17}$ This may have been because the training programmes had a rigid design. Better compliance was reported in a l year study of an "individually formed exercise programme" in 14 adolescents. ${ }^{11}$ Unsupervised exercise training may be better maintained by adults; two studies of home cycle ergometry training have shown positive results. ${ }^{713}$

If patients with CF are to benefit from long term exercise, a sustainable programme is needed which is based outside the hospital. Lack of health resources make it impossible to conduct long term supervised hospital based exercise training programmes. We have devised an individualised programme, inclusive of patient preferences, which would give the best chance of patients successfully incorporating regular exercise into their daily life and an already demanding treatment routine.

The individualised unsupervised programme of exercise training was studied in a randomised controlled trial in adults with CF over 1 year. We hypothesised that exercise training would result in increased fitness as measured by submaximal exercise testing. The primary outcome measure was whole blood lactate levels. This measurement was chosen because it is reproducible and removes psychological factors from the test. Secondary outcome measures were heart rate and, because of previous reports in children, ${ }^{14}$ we wanted to assess whether there was slowing of the rate of decline or stabilisation of lung function with long term exercise.

\section{METHODS}

\section{Patients}

Patients with CF who were willing to participate were recruited from a population of 150 attending the adult CF centre in Manchester at the time of the study. All subjects

Abbreviations: $\mathrm{BMI}$, body mass index; $\mathrm{CF}$, cystic fibrosis; $\mathrm{FEV}_{1}$, forced expiratory volume in 1 second; FVC, forced vital capacity; HR, heart rate; $\mathrm{RER}$, respiratory exchange ratio; $\mathrm{RR}$, respiratory rate; $\mathrm{SaO}_{2}$, oxygen saturation; $\mathrm{VE}$, ventilation; $\mathrm{VO}_{2}$, oxygen consumption. 
had documented CF on the basis of clinical history plus either an increased sweat chloride or abnormal genetic testing. Exclusion criteria were participation in another clinical trial, pregnancy, transplant listing, or clinical cor pulmonale. Ethical approval for the study protocol was obtained from the hospital's research ethics committee and subjects provided informed consent before entering the study.

\section{Protocol}

The subjects were randomised to either active or control groups in a ratio of three to two. A stratified randomisation in blocks was used to balance the groups for forced expiratory volume in l second $\left(\mathrm{FEV}_{1}\right)$, sputum colonisation by Burkholderia cepacia (both factors of known prognostic significance) and sex. The two groups were treated as follows.

\section{Active subjects}

The fitness of these subjects was first assessed as outlined below. They were also interviewed and completed a written questionnaire to establish their current exercise habits and preferences. Based on this information, a plan to increase the physical activity of each subject was formulated with particular emphasis on their preferences. The aim was for the subjects to engage in a lower/whole body aerobic exercise programme (such as walking/jogging, cycling, swimming, playing a sport) and an upper body exercise (usually weight training and based at home or at a gym). The subjects participated fully in the discussion and the investigator sought to find ways of facilitating and motivating the subject. The upper body component tended to be more prescriptive. Material interventions included supplying home exercise equipment such as weights or arranging free access to local sports facilities.

Once the activities had been decided upon, a training schedule was defined. The aim was to achieve three sessions of upper body exercise and three of lower body exercise per week. Each session lasted approximately 20 minutes and subjects were allowed to combine the upper and lower body sessions on one day if this was more practical. Each session started with a warm up period followed by the training exercise, after which a warm down period was advised.

Starting work intensities (based on fitness and disease severity) and duration were advised depending on the results of the initial assessment and a plan of how to progress was defined. Perceived work intensity scores were used to guide subjects to exercise at an adequate training intensity. In most cases an initial physiotherapist supervised training session was needed; this was always the case for weight training. The importance of warm up was emphasised and attention paid to starting posture and correct technique to avoid injury. The starting weight was one which could be lifted comfortably 10-15 times; this progressed to 20-30 times before increasing the sets and then the weight. The majority of subjects used weight training as their upper body exercise and were directed to a predominantly aerobic programmenamely, lower weights and greater repetitions. Equipment (weights and a bench) was provided for subjects who were exercising at home and subjects were taught the individual exercises and taken through an initial training session. A written protocol was provided which specified how to progress through increased repetitions, sets, and then loads.

The active subjects were seen at 4 week intervals for discussion of progress, encouragement, and for the training schedule to be advanced. Flexibility to change the programme was allowed. Subjects were asked to keep a training diary and to bring this with them at all visits to aid progression of the training intensity.

\section{Control subjects}

Control subjects were not asked to alter their activity levels in any way although, if they expressed a desire to do more exercise, this was not discouraged. In all aspects apart from the exercise intervention they were treated in an identical fashion to the active group.

All subjects continued their usual treatment regimens and routine clinical review was not altered by their participation in the study. In the event of a disease exacerbation, subjects in both groups were treated by the multidisciplinary team for their specific medical problem. When patients were acutely unwell or febrile they were instructed not to exercise and, once in recovery, those in the active group were advised a "holding" programme of lower intensity. If subjects were due for an assessment while unwell, this was delayed until they had recovered.

\section{Assessments}

All subjects had a baseline assessment, were monitored at 3 and 6 months, and results for the active and control group were compared at 12 months. The assessments took place on a single day and were the same except that the baseline assessment was preceded by a single visit for maximal exercise testing so that the peak exercise capacity was known. This information was used to decide the workloads to be used on submaximal testing (see later) and also to aid the prescription of exercise.

Spirometric parameters were measured before exercise testing using a dry bellows spirometer (Vitalograph, Buckingham, UK) and the best of three attempts was recorded. FEV $_{1}$ and forced vital capacity (FVC) were measured in litres and also expressed as a percentage of predicted normal values. ${ }^{18}$

Height and weight were measured at each visit with shoes and outerwear removed. Body mass index (BMI) was calculated as weight/height ${ }^{2}$.

\section{Exercise tests}

Subjects performed both cycle ergometry and arm ergometry exercise tests. Submaximal (constant work rate) tests were used for the purposes of assessment. A constant work rate test was chosen because it more closely represented the activities of daily living. This technique has previously been used in patients with chronic obstructive pulmonary disease to demonstrate increased fitness following training. ${ }^{19}$ In addition to the physiological measurements, changes in the perceived intensity of exercise symptoms following training were examined.

Before their first assessment, subjects attended on a separate day for maximal exercise testing. Subjects were reviewed and performed spirometric tests before exercise testing in order to confirm clinical stability. The measurement equipment used was the same for all exercise protocols. Bicycle and arm ergometric tests were performed using electromagnetically braked ergometers (Rodby Electronic RE830; Sodertalje, Sweden) adjusted to suit the subject before testing.

Pulmonary gas exchange was determined using a computerised breath by breath analyser (CPX, Medical Graphics, Minnesota, USA). Arterial oxygen saturation $\left(\mathrm{SaO}_{2}\right)$ was measured continuously by pulse oximetry (Biox 7300a, Ohmeda, Kentucky, USA) as was the heart rate (HR-S and $\mathrm{W}$, Denmark). The predicted peak heart rate $\left(\mathrm{HR}_{\text {peak }}\right)$ was calculated from age $(210-2 / 3$ age $)$ and the predicted peak oxygen consumption $\left(\mathrm{Vo}_{2 \text { peak }}\right)$ was calculated from age, sex, height, and weight. ${ }^{20}$ The respiratory exchange ratio (RER) was calculated as $\mathrm{VCO}_{2} / \mathrm{VO}_{2}$. 
Immediately after the end of exercise subjects were asked to score their sense of "breathlessness" and "muscle effort/ fatigue" at peak exercise using Borg scales. ${ }^{21}$

Whole blood lactate was measured at the end of exercise from a finger prick capillary sample taken 1 minute after the end of exercise. An immediate lactate analysis was performed using an enzymatic method (Analox GM7, Hammersmith, London, UK).

\section{Symptom limited maximal exercise tests}

Maximal exercise tests were performed before the baseline assessment. Subjects were initially familiarised with the exercise equipment and symptom scoring. They then performed progressive exercise to a symptom limited maximum using the electromagnetically braked bicycle ergometer. A ramp protocol was used with an incremental work rate of 8-25 W/min judged by the operator according to disease severity and likely fitness, with the aim for a test lasting approximately 8 minutes. Subjects were encouraged to make a maximal effort and continued until limited by symptoms or until they could no longer maintain a pedalling rate of $50 \mathrm{rpm}$. After a minimum interval of 1 hour they then performed a maximal arm ergometric test. The protocol was the same except that the incremental rate was chosen in the range of $5-12 \mathrm{~W} / \mathrm{min}^{22}$ The peak exercise measurements were made as described above and recorded as the mean from the final eight breaths before finishing exercise. The peak exercise variables included: heart rate (HR), ventilation $(\mathrm{VE})$, work load (Work), respiratory rate (RR), oxygen saturation $\left(\mathrm{SaO}_{2}\right)$, whole blood lactate, and Borg scores of "breathlessness" and "muscle fatigue/effort". The peak workload obtained on the maximal tests was used to determine the workloads used in the submaximal constant load tests described below.

\section{Constant work rate (submaximal) exercise tests}

On each assessment visit the subjects performed a constant work rate bicycle ergometry test and then, after a minimum interval of 1 hour, went on to constant work rate arm ergometry testing. The submaximal tests were repeated at each assessment using exactly the same workloads on each occasion.

Subjects first warmed up with 1 minute of unloaded pedalling and then 4 minutes at a workload equivalent to $40 \%$ of the peak workload from their maximal test. The workload was then increased to $55 \%$ of peak for a further 6 minutes. ${ }^{19}$ This intensity was chosen to give a lactate measure above the anaerobic threshold but not too high to cause exhaustion. Measurements were made as before with the outcome variables recorded as the mean value over the final minute of exercise. Borg scales and lactate measurements were made at the end of exercise as for the maximal tests.

\section{Statistical analysis}

Subjects were analysed on an intention to treat basis. Missing data points were treated by omission rather than

\begin{tabular}{lll}
\multirow{2}{*}{$\begin{array}{l}\text { Table } 1 \\
\text { for active }(n=30) \text { and control subjects }(n=18)\end{array}$} \\
\hline
\end{tabular}

interpolation. Statistical analysis was performed using SPSS for Windows version 6 (SPSS Inc, Chicago, IL, USA).

An initial power study performed on the patient group (31 active subjects and 20 controls) to detect changes in lactate levels showed the study would have an $80 \%$ power to detect changes of $0.7 \mathrm{mmol} / \mathrm{l}$ or greater between the two groups assuming a common standard deviation of 0.9 and a comparison using the simple $t$ test with the conventional 5\% significance level.

Comparisons between the active and control subjects were made using unpaired $t$ tests for normally distributed variables and Mann-Whitney U tests for the remainder. Variables were compared at 12 months as change from baseline. A two tailed $p$ value of $<0.05$ was taken to indicate statistical significance.

\section{RESULTS}

\section{Baseline data}

Fifty one subjects (31 active and 20 controls) were randomised. One subject withdrew consent and two were withdrawn. One patient in the active group was withdrawn for failure to attend the initial assessment appointment and two of the control group were withdrawn (one because of prolonged ill health at the time of the initial assessment visit and the other following a subarachnoid haemorrhage secondary to an unsuspected arteriovenous malformation). Forty two patients completed the 12 month programme. Every effort was made to obtain data but patients failed to attend for the tests or were too ill; one further patient was unable to complete the arm ergometry due to hand fractures. The main indicators of health status in adults with CF are nutrition and pulmonary function. A review of all adults with $\mathrm{CF}$ attending the clinic at the time of the study showed a mean $\mathrm{FEV}_{1}$ of $2.36 \mathrm{l}$, a mean BMI of $21.6 \mathrm{~kg} / \mathrm{m}^{2}$, and a mean age of 22.9 years, which are similar findings for the study patients.

Table 1 shows summary comparisons of the non-exercise measurements at baseline and table 2 shows comparisons between the active and control groups at baseline for maximal bicycle and arm ergometry. The subjects made a good overall effort as evidenced by peak heart rate, RER, and peak lactate levels. The median Borg breathlessness score was exceeded by that for muscle effort which was perceived as between "severe" and "very severe". A similar pattern of responses was seen for peak arm ergometry as with bicycle ergometry, but with some reduction in measured values of mean peak oxygen uptake and mean peak heart rate. The ratios of leg to arm variables are consistent with those recorded in previous studies of arm exercise in normal individuals. ${ }^{22}$ Mean peak ventilation and blood lactate levels were similarly lower for arm exercise. Subjects again perceived muscle effort in excess of dyspnoea. The muscle effort scores were almost identical to those recorded with leg exercise but dyspnoea scores were lower.

Table 3 shows comparisons between the active and control groups at baseline for the constant work rate bicycle and arm ergometric tests. Subjects were working at quite high intensities during this test so had moderately high mean heart rates, respiratory rates, and ventilation. The mean lactate concentrations confirm that subjects had a moderately high intensity of muscle effort and were working above the "lactate threshold" (by convention usually defined as $2 \mathrm{mmol} / \mathrm{l}$ ). These mean values were therefore at concentrations where changes in fitness would be expected to result in a change in the measured lactate level. The median Borg symptom score for muscle effort for cycle and arm ergometry equated to a subjective rating of "somewhat severe". The median Borg breathlessness scores for cycle and arm ergometry were close to a subjective rating of "moderate". 
Table 2 Peak exercise data for active $(n=30)$ and control group subjects $(n=18)$

\begin{tabular}{|c|c|c|c|c|}
\hline & \multicolumn{2}{|c|}{ Bicycle ergometry } & \multicolumn{2}{|c|}{ Arm ergometry } \\
\hline & Active & Control & Active & Control \\
\hline Work $_{\text {peak }}$ (W) & $142(51.0)$ & $154(41.0)$ & $85.8(22.9)$ & $83.3(20.9)$ \\
\hline Lactate (mmol/I) & 6.5 (1.8) & $7.3(1.8)$ & $5.5(1.6)$ & $6.0(2.1)$ \\
\hline $\mathrm{HR}_{\text {peak }}$ (bpm) & $170(13.2)$ & $166(9.7)$ & $157(16.2)$ & $155(19.3)$ \\
\hline RER & $1.22(0.08)$ & $1.23(0.08)$ & $1.16(0.10)$ & $1.21(0.09)$ \\
\hline$V_{E_{\text {peak }}}(1 / \mathrm{min})$ & $65.5(21.3)$ & 69.4 (18.4) & $52.0(17.0)$ & $52.0(16.6)$ \\
\hline $\mathrm{RR}_{\text {peak }}$ (breaths/min) & $41.2(9.6)$ & $39.4(8.4)$ & $43.9(9.2)$ & 40.6 (11.7) \\
\hline $\mathrm{SaO}_{2 \text { peak }}(\%)$ & 94.5 (3.5) & $94.5(2.4)$ & $93.0(3.8)$ & $94.6(2.7)$ \\
\hline \multicolumn{5}{|l|}{ Borg scores } \\
\hline Breathlessness & $4.5(0-7.5)$ & $3.75(1-7)$ & $3.0(0.5-8)$ & $3.0(0-7)$ \\
\hline Muscle effort & $7.0(1.5-10)$ & $6.0(3-10)$ & $5.0(2-10)$ & $7.0(3-10)$ \\
\hline
\end{tabular}

For all variables the active and control groups had similar baseline characteristics.

\section{Change in the non-exercise variables (pulmonary function)}

Changes in mean values from baseline for spirometric parameters and BMI are shown in table 4. There was a clear trend to better preservation of pulmonary function in the active group. The $\mathrm{FEV}_{1}$ in the active group was essentially unchanged at 12 months and was only 67 (63) ml lower than baseline. The control group at 12 months had a larger fall in $\mathrm{FEV}_{1}$ with a reduction of 174 (57) $\mathrm{ml}$ from baseline. There was an improvement in FVC of 46 (72) $\mathrm{ml}$ in the active group compared with a deterioration in the control group of -167 (68) $\mathrm{ml}(\mathrm{p}<0.05)$.

\section{Change in constant work rate exercise performance Cycle ergometry}

The changes in constant work rate bicycle exercise performance are shown in table 5 . The main differences between the two groups were significant reductions in the lactate concentrations $(p<0.05)$ and heart rates $(p<0.05)$ in the active group compared with control subjects at 12 months, indicating a training effect in the active group.

\section{Arm ergometry}

The changes in constant work rate arm ergometric performance are shown in table 6. A small but non-significant reduction in the lactate concentration was observed in the active group at 12 months. In addition, there was a small reduction in ventilation in the active group.

\section{DISCUSSION}

This study is the first in adults with CF to examine the response to home based, individualised, unsupervised exercise training in the setting of a randomised controlled trial. In the long term it has shown improved fitness by comparing changes in lactate levels between the active and control groups as the primary outcome measure over a 1 year period and supports the hypothesis that a training effect (as measured by reduction in heart rate) can be achieved with this type of programme. Importantly, as a secondary outcome there was preservation of lung volumes in the training group compared with controls. This study complements a recent 3 year study of a home exercise programme for children and adolescents with CF with less severe disease. ${ }^{14}$ Similar findings were reported in that study where a reduced decline in FVC was recorded in exercising subjects compared with controls but without any increase in exercise capacity. The only previously published randomised controlled trial of exercise in CF examined the response to supervised exercise training over a maximum of 14 days in patients being treated in hospital for an exacerbation of their pulmonary disease. ${ }^{23}$ Previous studies of exercise in CF have predominantly been of small numbers, often uncontrolled, and of less than 3 months duration. These studies have shown that patients with CF can increase their exercise capacity with exercise training, although the best results have come from studies involving supervised training. ${ }^{6}{ }^{24}$

For bicycle ergometry the primary outcome measurement of fitness was the lactate concentration at the end of the constant work rate test. The active group showed a decrease in the mean lactate level for leg exercise at 12 months, thus indicating that a physiological training effect had occurred. Improved fitness was also supported by the observation of a reduction in heart rate (training bradycardia). A reduction in heart rate at submaximal workloads during incremental testing has previously been reported following a 3 month programme of jogging which resulted in an increased peak exercise capacity. ${ }^{6}$ The reduction in lactate that we recorded

Table 3 Summary submaximal test data at baseline for active $(n=30)$ and control group subjects $(n=18)$

\begin{tabular}{|c|c|c|c|c|}
\hline & \multicolumn{2}{|c|}{ Bicycle ergometry } & \multicolumn{2}{|c|}{ Arm ergometry } \\
\hline & Active & Control & Active & Control \\
\hline Work (W) & $80(28.5)$ & 85 (24.2) & 50 (13.6) & $51.0(11.6)$ \\
\hline Lactate (mmol/l) & $5.1(1.3)$ & $5.3(1.4)$ & $4.3(1.6)$ & $4.5(1.2)$ \\
\hline HR (bpm) & $152(12.7)$ & $147(13.6)$ & $135(14.4)$ & $129(17.5)$ \\
\hline RER & $1.07(0.06)$ & $1.07(0.04)$ & $1.06(0.06)$ & $1.08(0.05)$ \\
\hline$V_{E}(I / m i n)$ & 47.5 (13.0) & 50.7 (12.9) & $38.1(9.2)$ & $37.4(8.9)$ \\
\hline RR (breaths/min) & $32.4(6.6)$ & $31.8(8.0)$ & $35.7(8.5)$ & $33.8(7.6)$ \\
\hline $\mathrm{SaO}_{2}(\%)$ & $94.8(3.8)$ & $95.2(2.9)$ & $95.9(2.4)$ & $96.0(3.4)$ \\
\hline \multicolumn{5}{|l|}{ Borg scores } \\
\hline Breathlessness & $3.0(0-8)$ & $3.0(1-8)$ & $3.0(0-6.5)$ & $2.5(0-6)$ \\
\hline Muscle effort & $3.25(1-8)$ & $4.0(0.5-8)$ & $4.0(2-8)$ & $3.5(0.5-8)$ \\
\hline
\end{tabular}


Table 4 Comparison of changes from baseline to 12 months in non-exercise variables (mean values) for active and control group subjects

\begin{tabular}{lllll}
\hline & $\begin{array}{l}\text { Active } \\
(\mathbf{n}=25)\end{array}$ & $\begin{array}{l}\text { Control } \\
(\mathbf{n}=17)\end{array}$ & Mean (95\% Cl) change & p value \\
\hline $\mathrm{FEV}_{1}(\mathrm{ml})$ & -67 & -174 & $107(-74$ to 288$)$ & 0.24 \\
$\mathrm{FVC}(\mathrm{ml})$ & 46 & -167 & $213(3$ to 423$)$ & 0.048 \\
$\mathrm{BMI}\left(\mathrm{kg} / \mathrm{m}^{2}\right)$ & 0.30 & -0.24 & $0.54(-0.09$ to 1.17$)$ & 0.09 \\
\hline
\end{tabular}

at a submaximal workload suggests the potential for an increased peak exercise capacity. The changes in lactate and heart rate were significantly different from the control group which showed a decrease in fitness marked by small rises in lactate and heart rate.

For arm ergometry there was no difference in lactate levels at 12 months between the active and control groups. One explanation for this finding might be that the adherence to the upper body component of the programme was not sustained for 12 months, possibly because the upper body programme was more prescriptive than the lower body programme.

One marked difference between the arm and cycle ergometry results was the reduction in ventilation for arm exercise in the active group. Given that the workloads were identical, this observation probably resulted from greater efficiency in generating the required output. Arm ergometry would be a much less familiar form of exertion to the subjects than cycling, and therefore lends itself to subjects "learning" to perform more efficiently.

There was a clear trend to lower muscle effort scores in the active group for both leg and arm ergometry. This was probably a consequence of improved fitness but habituation might have been an additional component. The one study of the effect of exercise training on symptom perception in $\mathrm{CF}$ only examined dyspnoea. ${ }^{10}$ However, the authors found a decrease in breathlessness at a given submaximal minute ventilation despite there being no change in peak exercise capacity.

As a consequence of improved fitness and the trend to reduced symptoms at submaximal workloads, the subjects in the training group should be able to enjoy increased levels of exercise in their daily lives. This improvement would be expected to have quality of life benefits. Improved ability to perform activities of daily living and perceived competence (including positive feelings of self-worth and physical appearance) have previously been shown with exercise training in $\mathrm{CF}^{8}{ }^{8}{ }^{17}$

Pulmonary function (particularly $\mathrm{FEV}_{1}$ ) and body weight are the best objective measurements of disease severity and clinical outcome in $\mathrm{CF}^{25} \mathrm{FEV}_{1}$ is probably the single best measurement for the assessment of prognosis. ${ }^{26}$ Other clinical variables may add to any individual assessment but, in a recent multivariate analysis of data from a population of 635 patients, ${ }^{27}$ the only factors associated with the risk of death were the baseline $\mathrm{FEV}_{1}$ and its subsequent rate of decline. As pulmonary disease severity is the main factor determining the outcome in CF, any intervention which either improves or preserves pulmonary function in the long term would have fundamental implications for disease management and subsequent survival.

Several small uncontrolled studies of exercise training have included measurements of lung function. Improved $\mathrm{FEV}_{\mathrm{l}}$ was found with programmes of swimming ${ }^{16}$ and bicycle exercise, ${ }^{13}$ and reduced residual volume was reported with weight training ${ }^{9}$ and general exercise programmes. ${ }^{10}{ }^{12}$ Another study reported unchanged lung function over 12 months with individualised exercise. ${ }^{12}$ In addition, a 3 month study of running also found no change in active participants but a significant decline in pulmonary function was observed in the randomised controlled group. ${ }^{6}$ The validity of the results of these studies is limited by the small numbers of patients studied, short duration, and the absence of randomised controls. The benefit to pulmonary function is supported by a 3 year randomised controlled trial of individualised home exercise in 65 children and adolescents in which there was a significant reduction in the annual rate of decline of FVC and a trend to a lower rate of decline in $\mathrm{FEV}_{1}$ in those undertaking exercise. ${ }^{14}$ These benefits occurred despite there being no increase in fitness as measured by peak exercise testing.

Our study has considerable advantages in design. There was a clear trend to better preserved lung function in our active group with a decrease of only $67 \mathrm{ml}$ at 12 months. This mean fall in $\mathrm{FEV}_{1}$ at 1 year was $-1.5(1.4) \%$ predicted and is therefore at the lower range of previously published estimates of the rate of decline in pulmonary decline of $2-4 \%$ per year. ${ }^{28-30}$ In the control group the $\mathrm{FEV}_{1}$ had fallen by a mean of $-174 \mathrm{ml}$ at 12 months which equated to a fall of 4.4 (1.5)\% predicted. There was a small improvement in FVC in the active group over the year but a significant decline in FVC in the control group. The finding of an improvement in FVC is important to the individual patient and almost certainly translates into an improvement in functional capacity and therefore a greater ability to undertake some of the more exertional activities of daily living.

Table 5 Comparison of changes from baseline to 12 months in bicycle exercise performance (mean values) for active and control group subjects

\begin{tabular}{lllll}
\hline & $\begin{array}{l}\text { Active } \\
(\mathbf{n}=\mathbf{2 5})\end{array}$ & $\begin{array}{l}\text { Control } \\
(\mathbf{n}=\mathbf{1 7})\end{array}$ & Mean $(\mathbf{9 5 \%} \mathrm{Cl})$ change & p Value \\
\hline Lactate $(\mathrm{mmol} / \mathrm{l})$ & -0.38 & 0.45 & $-0.83(-1.53$ to -0.13$)$ & 0.02 \\
HR $(\mathrm{bpm})$ & -4.8 & 3.4 & $-8.2(-15.6$ to -0.8$)$ & 0.03 \\
RER & 0.00 & -0.02 & $0.02(-0.01$ to 0.05$)$ & 0.24 \\
VE (I/min) & -1.6 & 0.9 & $-2.5(-6.1$ to 1.1$)$ & 0.17 \\
RR (breaths/min) & 0.8 & 1.6 & $-0.8(-4.9$ to 3.3$)$ & 0.67 \\
$\begin{array}{l}\text { Borg scores } \\
\text { Breathlessness }\end{array}$ & 0 & 0 & $0(-1.0$ to 1.0$)$ & 0.86 \\
$\quad$ Muscle effort & 0 & 0 & $-0.3(-1.5$ to 0.9$)$ & 0.91 \\
\hline
\end{tabular}




\begin{tabular}{|c|c|c|c|c|}
\hline & $\begin{array}{l}\text { Active } \\
(n=23)\end{array}$ & $\begin{array}{l}\text { Control } \\
(n=16)\end{array}$ & Mean $(95 \% \mathrm{Cl})$ change & $p$ Value \\
\hline Lactate (mmol/l) & -0.28 & 0.04 & $-0.32(-1.15$ to 0.51$)$ & 0.43 \\
\hline HR (bpm) & -2.8 & -1.4 & $-1.4(-10.2$ to 7.4$)$ & 0.76 \\
\hline RER & -0.01 & -0.01 & $0(-0.04$ to 0.04$)$ & 0.92 \\
\hline VE $(I / \mathrm{min})$ & -1.9 & 1.4 & $-3.3(-6.4$ to -0.2$)$ & 0.04 \\
\hline RR (breaths/min) & 2.4 & 0.9 & $1.5(-3.1$ to 6.1$)$ & 0.49 \\
\hline \multicolumn{5}{|l|}{ Borg scores } \\
\hline Breathlessness & -1 & 0 & $-0.9(-1.9$ to 0.1$)$ & 0.07 \\
\hline Muscle effort & 0 & 0 & $0.3(-1.0$ to 1.6$)$ & 0.27 \\
\hline
\end{tabular}

There was a definite training advantage in the active group in this study, although the changes we observed were moderate in size. This may partly be because the intervention was designed with the emphasis on patient acceptability and thus the longer term adherence to exercise, rather than achieving the greatest possible increase in fitness for a specific activity.

Exercise training must be of adequate intensity to achieve a training effect and a greater physiological improvement is achieved at higher work rates, irrespective of the total amount of work performed..$^{19}$ We tried to guide our subjects towards moderate/higher training intensities and used work intensity scores to facilitate this. We also applied generally accepted guidelines regarding training duration and frequency to their individual programme. The active subjects were asked to perform at least three 30 minute sessions per week for both upper and lower body exercise. Clearly, close supervision is advantageous in achieving an optimal training effect, but this was impractical, not part of the trial design, and some subjects may have exercised at lower than optimal work rates.

A disadvantage of unsupervised exercise is that adherence is not easily monitored and adherence with a training programme of this nature might potentially be poor. Despite this concern, we felt that the individualised unsupervised approach was more practical for our population and may be more sustainable. Factors which made supervised training less suitable for our patient setting included the large geographical area covered, concerns about cross infection, arranging sessions around work or other commitments and, importantly, resource implications which would be difficult to maintain in the longer term.

It is unrealistic to expect $100 \%$ adherence with exercise over 12 months and the study was designed to examine response to a training intervention in a realistic clinical setting. The exercise diaries were intended to aid guidance of training intensity as well as exercise frequency but they were, with a few exceptions, poorly kept and infrequently returned at assessment periods as found in other studies. ${ }^{31}$

Clearly, the finding of a physiological training effect on exercise testing is evidence that training took place. Improvements in lactate levels and heart rate were observed over the full 12 months following cycle ergometric testing, which suggests reasonable adherence to the lower body aerobic exercise. This may have been facilitated by the individualised approach. In contrast, the magnitude of change in lactate levels for upper body exercise was small. This might result from the fact that the upper body aerobic weight training programme was much more uniform and "prescriptive" than the more varied/individualised lower body activities and thus might be less acceptable to the subjects.

Adherence to exercise has been recognised as a problem since the early studies of exercise in Zach et al found beneficial effects including improved lung function from a 7 week programme of supervised swimming in 11 children. ${ }^{16}$ However, most patients stopped exercise (only one continued) and lung function had returned to baseline levels 10 weeks after the programme ended. Adherence is a particular issue with unsupervised exercise as there is no external motivator. A 3 month study of home based exercise in 41 children noted that adherence decreased markedly towards the end of the study and concluded that this approach was of "limited value". ${ }^{15}$ The exercise intervention was, however, very prescriptive (adapted Royal Canadian Airforce exercises and running/jogging) and this may have contributed to the poor adherence. A study of 14 adolescents over 1 year reported better results from adopting an "individually formed exercise programme" based on patient preferences but, although $\mathrm{Po}_{2}$ improved, there was no improvement in fitness. ${ }^{11}$

A recent study examined home exercise cycle training for five sessions per week (one supervised) in 14 children. ${ }^{17}$ Greater leg muscle strength was observed although growth and increased body mass could have explained this observation. There was, however, only a small non-significant increase in peak exercise capacity and therefore adherence must be questioned. Most subjects expressed a willingness to continue some exercise at the end of the study but most stopped the cycling. They found the cycle training unacceptable and cited intensity and lack of variation as their main complaints.

We feel that the individualised nature of our programme enhanced patient acceptability and therefore increased activity levels. Another factor in favour of adherence in our study of home exercise is that adults might do better with unsupervised exercise. Improved performance has been reported with home cycle ergometry over 2 months in adults. ${ }^{7}$ Positive results were also apparent from a study of unsupervised home bicycle exercise over the year following an intensive 3 week inpatient rehabilitation programme in adult patients with severe disease..$^{13}$ Enhanced nutrition was part of the approach. This study showed that a multifaceted approach can achieve excellent results for patients with advanced disease.

In encouraging exercise one must allow for the conflicting aspects of self-care and time pressures in CF. Every effort must be made to adapt the exercise to fulfil the wishes of patients and to integrate it with their lifestyle. This study shows that benefit can be obtained with an individualised home based programme. In the long term, motivation must be sustained by the individual and the clinician must strive to engender an exercise habit. A flexible approach to encouraging exercise and an enthusiastic approach from the staff should not be underestimated. A feature that favours exercise adherence in CF is that the patients perceive it as an area over which they have control and that, unlike other treatments, fear of their disease does not drive adherence to exercise. ${ }^{32}$ 
Instead, they have a positive outlook on exercise regarding it as a "normal activity" which they can enjoy. In marked contrast to other treatments, CF patients actually underestimate their adherence with exercise ${ }^{33}$ and may be more open to encouragement.

In conclusion, this study has shown that a training effect can be achieved with a programme of individualised unsupervised home exercise in adults with CF over a 1 year period. Although intensive supervised programmes have achieved excellent short term results, a patient centred approach may have greater advantages in the longer term and offers a practical solution to encouraging exercise. Long term improvements in pulmonary function from sustained exercise are an ultimate goal which can potentially affect survival. ${ }^{34}$ Although neither this study in adults nor the recent study in children ${ }^{14}$ have conclusively answered the long term prognosis resulting from regular exercise, clear data have emerged which show that lung function is preserved in patients who exercise and is therefore a realistic objective of exercise training. CF teams should encourage and support exercise as a component of self-care. In contrast to other components of self-care, patients with CF enjoy exercise.

\section{Authors' affiliations}

A J Moorcroft, The Royal Shrewsbury Hospital, Shrewsbury SY3 8XQ, UK

M E Dodd, A K Webb, Manchester Adult CF Centre, South Manchester University Hospitals NHS Trust, Wythenshawe Hospital, Manchester M23 9LT, UK

J Morris, Wythenshawe Hospital, Manchester M23 9LT, UK

\section{REFERENCES}

1 Godfrey S, Mearns M. Pulmonary function and response to exercise in cystic fibrosis. Arch Dis Child 1971;46:144-51.

2 Cerny FJ, Pullano TP, Cropp, GJ. Cardiorespiratory adaptations to exercise in cystic fibrosis. Am Rev Respir Dis 1982;126:217-20.

3 Marcotte JE, Grisdale, RK, Levison H, et al. Multiple factors limit exercise capacity in cystic fibrosis. Pediatr Pulmonol 1986;2:274-81.

4 Coates AL, Boyce P, Muller D, et al. The role of nutritional status, airway obstruction, hypoxia, and abnormalities in serum lipid composition in limiting exercise tolerance in children with cystic fibrosis. Acta Pediatr Scand 1980;69:353-8

5 Lands LC, Heigenhauser GJ, Jones NL. Analysis of factors limiting maximal exercise performance in cystic fibrosis. Clin Sci 1992;83:391-7.

6 Orenstein DM, Franklin BA, Doershuk, et al. CF Exercise conditioning and cardiopulmonary fitness in cystic fibrosis. The effects of a three-month supervised running program. Chest 1981;80:392-8.

7 Salh W, Bilton D, Dodd $M$, et al. Effect of exercise and physiotherapy in aiding sputum expectoration in adults with cystic fibrosis. Thorax 1989;44:1006-8.
8 de Jong W, Grevink RG, Roorda RJ, et al. Effect of a home exercise training program in patients with cystic fibrosis. Chest 1994;105:463-8.

9 Strauss GD, Osher A, Wang Cl, et al. Variable weight training in cystic fibrosis. Chest 1987;92:273-6.

10 O'Neill PA, Dodds M, Phillips B, et al. Regular exercise and reduction of breathlessness in patients with cystic fibrosis. Br J Dis Chest 1987;81:62-9.

11 Blomquist M, Freyschuss U, Wiman LG, et al. Physical activity and self treatment in cystic fibrosis. Arch Dis Child 1986;61:362-7.

12 Andreasson B, Jonson B, Kornfalt R, et al. Long-term effects of physical exercise on working capacity and pulmonary function in cystic fibrosis. Acta Pediatr Scand 1987;76:70-5.

13 Heijerman HG, Bakker W, Sterk PJ, et al. Long-term effects of exercise training and hyperalimentation in adult cystic fibrosis patients with severe pulmonary dysfunction. Int J Rehabil Res 1992;15:252-7.

14 Schneiderman-Walker J, Pollock SL, Corey M, et al. A randomised controlled trial of a 3-year home exercise program in cystic fibrosis. J Pediatr 2000;136:304-10

15 Holzer FJ, Schnall R, Landau LI. The effect of a home exercise programme in children with cystic fibrosis and asthma. Aust Paediatr J 1984;20:297-301.

16 Zach MS, Purrer B, Oberwaldner B. Effect of swimming on forced expiration and sputum clearance in cystic fibrosis. Lancet 1981;ii:1201-3.

17 Gulmans VA, de Meer K, Brackel HJ, et al. Outpatient exercise training in children with cystic fibrosis: physiological effects, perceived competence, and acceptability. Pediatr Pulmonol 1999;28:39-46.

18 Knudson RJ, Lebowitz MD, Holberg CJ, et al. Changes in the normal maximum expiratory flow-volume curve with growth and aging. Am Rev Respir Dis 1983;127:725-34.

19 Cassaburi R, Patessio A, lolli $\mathrm{F}$, et al. Reductions in exercise lactic acidosis and ventilation as a result of exercise training in patients with obstructive lung disease. Am Rev Respir Dis 1991;143:9-18

20 Jones NL. Clinical exercise testing. Philadelphia: WB Saunders, 1988.

21 Borg GAV. Psychophysical basis of perceived exertion. Med Sci Sports Exerc 1982;14:377-81.

22 Franklin BF. Exercise testing, training and arm ergometry. Sports Med 1985;2:100-19.

23 Cerny FJ, Cropp, GJ, Bye MR. Hospital therapy improves exercise tolerance and lung function in cystic fibrosis. Am J Dis Child 1984;138:261-5.

24 Edlund LD, French RW, Herbst JJ, et al. Effects of a swimming program on children with cystic fibrosis. Am J Dis Child 1986;140:80-3.

25 Mahadeva R, Webb K, Westerbeek RC, et al. Clinical outcome in relation to care in centres specialising in cystic fibrosis: cross-sectional study. BMJ 1998;316:1771-5

26 Kerem E, Reisman J, Corey M, et al. Prediction of mortality in patients with cystic fibrosis. N Engl J Med 1992;326:1187-91.

27 Milla CE, Warwick WJ. Risk of death in cystic fibrosis patients with severely compromised lung function. Chest 1998;113:1230-4.

28 Corey M, Levison H, Crozier D. Five to seven year course of pulmonary function in cystic fibrosis. Am Rev Respir Dis 1976;114:1085-92.

29 Lewin LO, Byard PJ, Davies PB. Effect of Pseudomonas cepacia colonisation on survival and pulmonary function of cystic fibrosis patients. J Clin Epidemiol 1990;43:125-31.

30 Penketh ARL, Wise A, Mearns MB, et al. Cystic fibrosis in adolescents and adults. Thorax 1987;42:526-32.

31 Cote J, Cartier A, Malo J. Compliance with peak expiratory flow monitoring in home management of asthma. Chest 1998;113:968-72.

32 Abbott J, Dodd M. Webb AK. Health perceptions and treatment adherence in adults with cystic fibrosis. Thorax 1996;51:1233-8

33 Abbott J, Dodd M, Bilton D, et al. Treatment compliance in adults with cystic fibrosis. Thorax 1994;49:115-20.

34 Nixon PA, Orenstein DM, Kelsey SF, et al. The prognostic value of exercise testing in patients with cystic fibrosis. N Engl J Med 1992;327:1785-8. 